\title{
SYNERGY OF LIDAR AND PASSIVE REMOTE SENSOR DATA FOR RETRIEVING PROFILES OF MICROPHYSICAL PROPERTIES OF NON-SPHERICAL PARTICLES
}

\author{
Alexei Kolgotin $^{1 *}$, Detlef Müller ${ }^{2,3}$, Eduard Chemyakin ${ }^{3}$, Anton Romanov ${ }^{4}$ \\ ${ }^{I}$ Physics Instrumentation Center, Troitsk, Moscow Region, 142190, Russia, *alexeift@yahoo.com \\ ${ }^{2}$ University of Hertfordshire, Hatfield, Hertfordshire, AL10 9AB, UK, d.mueller@herts.ac.uk \\ ${ }^{3}$ Science Systems and Applications, Inc. (SSAI), NASA LaRC, \\ 1 Enterprise Parkway, Hampton, VA, 23666, USA, \\ ${ }^{4}$ The National University of Science and Technology, Leninskii av. 4, Moscow, 119049
}

\begin{abstract}
In this study we explore how the combination of 3 backscatter and 2 extinction lidar data with data that can be collected with ground-based and space-borne passive remote sensors, e.g. phase function coefficients which can be derived at various measurement wavelengths and scattering angles can result in improved profiles of particle microphysical properties. The algorithm is based on a light-scattering model that uses a mixture of spheres and randomly oriented spheroids.
\end{abstract}

\section{INTRODUCTION}

Our study addresses the question on what combination of lidar and passive remote sensing data could provide an optimum set of data for the retrieval of profiles of key particle parameters when observed from ground or space. Over the past 10 years, multiwavelength Raman/HSRL lidars have successfully demonstrated their capacity with regard to retrieving particle microphysical parameters $[1,2]$. In these approaches three backscatter $(\beta)$ and two extinction $(\alpha)$ coefficients measured with lidar at wavelengths $(\lambda)$ from 355 to $1064 \mathrm{~nm}$ are used as input data for the retrieval of particle size distribution (PSD) as well as particle bulk parameters, i.e. effective radius, number, surfacearea and volume concentrations, and the complex refractive index $m=m_{\mathrm{R}}-i m_{\mathrm{I}}$ (CRI). The major challenge in identifying the correct solution of the underlying ill-posed inverse problem is the limited number of input data, i.e. backscatter coefficients at 355, 532, and $1064 \mathrm{~nm}$, and extinction coefficients at 355 and $532 \mathrm{~nm}(3+2$ data), high measurement errors of up to $10-20 \%$, the wide range of particle radii $r$ from 0.05 to $15 \mu \mathrm{m}$, real parts $\left(m_{\mathrm{R}}\right)$ of the CRI from 1.3 to 1.6 and imaginary parts $\left(m_{\mathrm{I}}\right)$ from $i 0$ to $i 0.05$. Considering all these factors, it has been shown that different solution techniques result in large retrieval uncertainties of profiles of particle properties.

One of the ways that allows us to improve the retrieval quality is to increase the number of input data. In that context passive remote sensors provide us with valuable additional information. In this study we explore how the combination of $3+2$ lidar data with data that can be collected with ground-based and space-borne passive remote sensors, e.g. phase function coefficients which can be derived at various measurement wavelengths and scattering angles can result in improved profiles of particle microphysical properties. The algorithm is based on a light-scattering model that uses a mixture of spheres and randomly oriented spheroids. This light-scattering model is widely used in the analysis of passive remote sensing of particles of arbitrary shape, e.g. smoke, mineral dust, volcanic ash, and anthropogenic pollution.

\section{METHODOLOGY}

\subsection{Mathematical model}

The equation that is based on a light-scattering model that uses a mixture of spheres and randomly oriented spheroids and relates the PSD and its optical characteristics is presented in Ref. [3]. We can modify this equation as

$$
\begin{gathered}
\int_{r_{\min }}^{r_{\max }} k_{g}^{(\eta, \varepsilon)}(\lambda, m(z), r) v(r, z) d r=g(\lambda, z), \\
g=\alpha, \beta
\end{gathered}
$$

for the case of a height-dependent volume particle size distribution $v(r, z)$ and optical profiles $3 \beta(\lambda, z)+2 \alpha(\lambda, z)$, where the kernels of the integral equation 


$$
\begin{array}{r}
k_{g}^{(\eta, \varepsilon)}(\lambda, m(z), r)=(1-\eta) k_{g}(\lambda, m(z), r, \varepsilon=1)+ \\
+\eta k_{g}(\lambda, m(z), r, \varepsilon)(2)
\end{array}
$$

depend on particle shape $(\varepsilon)$ and the volume fraction of the spheroid particles $\eta \in[0 ; 1]$. If the particles are spherical the respective kernel $k_{g}(\ldots, \varepsilon=1)$ is calculated on the basis of Miescattering theory [4]. The kernels for randomly oriented spheroids $k_{g}(\ldots, \varepsilon)$ can be computed in the way shown in Ref. [5]. In our study we assume that the aspect ratio $\varepsilon$ (or aspect ratio distribution) in the sphere-spheroid model $(\eta, \varepsilon)$ is known, but that the spheroid fraction needs to be estimated.

Our goal is to find the profile $v(r, z)$ and the profile $m(z)$, see Eq. (1), on the radius and height domains $r \in\left[r_{\min } ; r_{\max }\right]$ and $z \in\left(0 ; z_{\max }\right]$ respectively. This is an ill-posed, underdetermined problem. In order to redefine this problem we include in the solution procedure data from a potential passive sensor that provides phase function coefficients $\varphi_{\theta}(\lambda)$ measured at 5 scattering angles $\theta=5^{\circ}, 30^{\circ}$, $60^{\circ}, 90^{\circ}$, and $120^{\circ}$ at 1 or 2 wavelengths, e.g. at $\lambda=410$ and/or $670 \mathrm{~nm}$. Passive sensors provide column-integrated data. Therefore, we have to reformulate Eq. (1) so that it can be used for column-integrated data as well. The integration of Eq. (1) over height $z$ allows us to find the equation that is consistent for data provided by both types of instruments

$$
\int_{r_{\min }}^{r_{\max }} k_{g}^{(\eta, \varepsilon)}(\lambda, m, r) v(r) d r=g(\lambda), g=\alpha, \beta, \varphi_{\theta}
$$

where

$$
\begin{gathered}
g(\lambda)=\int_{0}^{z_{\max }} g(\lambda, z) d z, v(r)=\int_{0}^{z_{\max }} v(r, z) d z, \\
g=\alpha, \beta
\end{gathered}
$$

and the parameter $m$ is equal to some intermediate value in the profile $m(z)$, so that the equation

$$
\begin{array}{r}
\int_{0}^{z_{\max }} \int_{r_{\min }}^{r_{\max }} k_{g}^{(\eta, \varepsilon)}(\lambda, m(z), r) v(r, z) d r d z= \\
\int_{r_{\min }}^{r_{\max }} k_{g}^{(\eta, \varepsilon)}(\lambda, m, r) \int_{0}^{z_{\max }} v(r, z) d z d r
\end{array}
$$

is fulfilled on the basis of the average theorem.

We use Eq. (3) and (1) in our strategy in terms of two steps in order to find the particle microphysical parameters for 1) the column integrated data, and 2) the profile data, taking into account the column-integrated data as benchmark.

\subsection{Retrieval approach for column- integrated data}

Eq. (3) for column-integrated data is the classical integral equation of the Fredholm type of the $1^{\text {st }}$ kind with unknown input parameters $\eta$ and $m$. This equation can be solved by inversion with regularization, as shown in Ref. [3], but now data from a passive sensor $(5-10 \varphi)$ are added to the input lidar data $(3 \beta+2 \alpha)$. It means that the underlying inversion problem (3) has $\mathrm{N}_{\mathrm{O}}=10-15$ pieces of input information, i.e., $3 \beta+2 \alpha+(5-10) \varphi$. Such a relatively high number of input data allows us to modify the algorithm in Ref. [3] and take into account just a few inversion windows $\left[r_{\min }, r_{\max }\right]$ located from 0.05 to $10-15 \mu \mathrm{m}$ particle radius. The use of 5-7 inversion windows instead of 100-120 inversion windows reduces the solution space by one order of magnitude.

We find the column-integrated PSD $v(r)$ as well as effective radius $r_{\text {eff }}$, number $n$, surface-area $s$ and volume $v$ concentrations, and the complex refractive index $m$. In section 3 we mainly describe this approach and show results of numerical simulations.

\subsection{Retrieval approach for profile data}

Eq. (1) can be considered as a system of Fredholm integral equations of the $1^{\text {st }}$ kind at a finite number of height bins $z_{l}, l=1,2, \ldots, \mathrm{N}_{\mathrm{L}}$, distributed along the height from 0 to $z_{\max }$. In a recent publication [6] the gradient correlation method (GCM) and proximate analysis (PA) were presented. GCM and PA allow us to find vertical profiles that contain stable solutions of the inversion problem. GCM and PA use regression equations that connect the lidar data products (optical data) and the particle microphysical parameters. It was shown in Ref. [6] that the careful estimation of the regression coefficients (RC) of the underlying regression equations permits for a comparably accurate retrieval of the PSD $v\left(r, z_{l}\right)$ as well as the profiles of effective radius $r_{\text {eff }}\left(z_{l}\right)$, number $n\left(z_{l}\right)$, surface-area $s\left(z_{l}\right)$ and volume $v\left(z_{l}\right)$ concentrations, and the complex refractive index $m\left(z_{l}\right)$. Knowledge of column-integrated data derived in step 1 of our strategy provides us with a robust estimation of the RCs. Detailed results of numerical simulation with GCM and PA can be found in Ref. [6]. 


\section{RESULTS OF NUMERICAL SIMULATIONS}

In our numerical simulations we generated synthetic optical data $3 \beta+2 \alpha+10 \varphi$. We used two types of PSDs, i.e. one monomodal and one bimodal PSD. The monomodal PSD (MPSD) is described by the log-normal law with mean radius $r_{0}=0.1 \mu \mathrm{m}$ and mean width $\sigma=1.49$. The bimodal PSD (BPSD) includes the fine mode with the same parameters $r_{0}$ and $\sigma$ of the MPSD and the coarse mode with $r_{0}=1 \mu \mathrm{m}$ and $\sigma=1.8$.

We use three values (levels) of particle lightabsorption. For low light-absorbing (LA) particles the CRI is $m=1.38-i 0.002$, for moderate lightabsorbing (MA) $m=1.45-i 0.005$, and for high light-absorbing (HA) $m=1.57-i 0.018$. Last but not least we use three types of sphere-spheroid models $(\eta, \varepsilon)$, i.e., $(0,0.33),(0.7,0.33)$ and $(0.75,033)$.

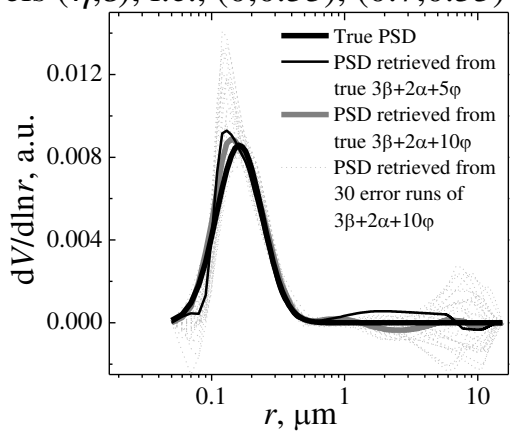

Fig. 1. Black thick curve shows the true $(0.7,0.33)-H A-$ MPSD. The grey solid curve and the 30 grey-dotted curves represent the PSDs retrieved in scenario $A$ and $C$, respectively, from the optical data set $3 \beta+2 \alpha+10 \varphi$. The black thin curve shows the PSD retrieved in scenario A from the optical data set $3 \beta+2 \alpha+5 \varphi$.

We generated two data sets, i.e., $3 \beta+2 \alpha+5 \varphi$ and $3 \beta+2 \alpha+10 \varphi$ that were used as input for the inversion problem (3). The solution space in the retrieval approach is defined on the basis of the input data that are defined by Eq. (3) as follows:

- 7 inversion windows on the radius domain $r_{\text {min }}=0.05-0.10 \mu \mathrm{m}$ and $r_{\max }=8-15 \mu \mathrm{m}$;

- 6 real parts of the CRI, equidistantly distributed between 1.35 and 1.6;

- 11 imaginary parts of the CRI, equidistantly distributed between $i 0$ and $i 0.05$;

- 5 spheroid fractions, equidistantly distributed between 0 and 1 .

We assume the following scenarios in our simulations in order to solve the inversion problem (3):
A. The optical data $3 \beta+2 \alpha+5 \varphi$ and $3 \beta+2 \alpha+10 \varphi$ are error-free; the spheroid fraction $\eta$ is unknown. B. The synthetic optical data $3 \beta+2 \alpha+(5-10) \varphi$ have maximum errors up to $5 \%$ and average errors up to $2 \%$; the spheroid fraction $\eta$ is not known.

C. the synthetic lidar data $(3 \beta+2 \alpha)$ have errors up to $10 \%$, the synthetic data of the passive sensor $(10 \varphi)$ have errors up to $5 \%$; the spheroid fraction $\eta$ is not known.

D. We use scenario $\mathrm{C}$ but the spheroid fraction $\eta$ is known.

We carried out inversions with noisy data (erroneous data), which implies that 30 different error runs were made in scenarios B-D.

We show the statistics of the results of our numerical simulations in Fig. 1-2 and Table 1-2. The results derived using scenario A agree well with the theoretical values. MPSD (thin black curves and BPSD (solid grey curves) in Fig. 1, 2 almost coincide, however the PSD is more stable in the case of $3 \beta+2 \alpha+10 \varphi$ (solid grey curve).

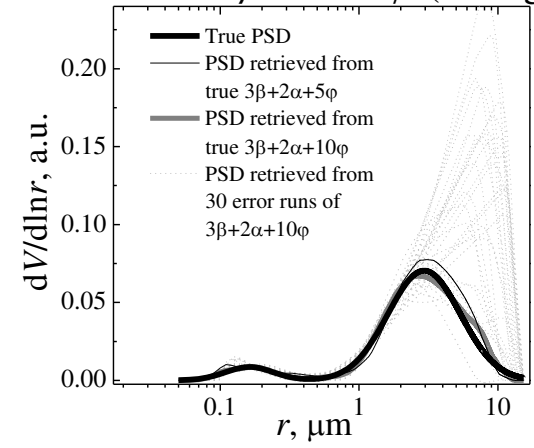

Fig. 2. The same as Fig. 1 but for (0.7,0.33)-HA-BPSD.

The uncertainties of the estimated parameters stay within a few percent. Such a quality of retrieval results cannot be obtained from a $3+2$ data set even if particle depolarization coefficients are available [3]. The uncertainties of the estimated real and imaginary parts do not exceed 0.025 and $i 0.0025$, respectively, which are half of the step sizes of our entry values, and which was never obtained in the case of using $3+2$ data only [3].

The results derived from scenario $\mathrm{B}$ are close to the results obtained in scenario A. The uncertainties of the estimated parameters of the PSD do not exceed $50 \%$ in the worst case. The CRI is estimated with the same uncertainty in both scenarios (not shown here).

The retrieval results become worse in scenario C. For some error runs we find that the PSDs show a 
strong (extensive) coarse mode (see dotted lines in Fig. 2). Such outliers result in the overestimation of the volume concentration and the effective radius of up to $155 \%$ and $120 \%$, respectively (see Table 1). The analysis of these PSDs shows that they are linked to very high values of the retrieved imaginary part, exceeding the true value by $i 0.03$ and more (see for example HA-MPSD in table 2). Simultaneously, the value of the spheroid fraction cannot be retrieved and we obtain the value 0 . It means that the sphere-spheroid model $(\eta, \varepsilon)$ can be mimicked by a pure sphere model $(0, \varepsilon)$ at the cost of overestimating the true values of the imaginary part and the volume concentration. We note that the retrieval of number concentration is more stable; the uncertainty is $80 \%$ whereas using the $3+2$ data set the uncertainty is $100 \%$ and above [3]. Surface-area concentration is the most stable parameter in the retrieval (uncertainty is 53\%). As we mentioned before, the uncertainty of the CRI retrieval increases as well; it is up to $i 0.04$ for the imaginary part and up to 0.07 for the real part. However, our approach still allows us to distinguish the L-MA and HA particles because in the first case the uncertainty is less than $i 0.009$ for the imaginary part of large particles (see LA, HA BPSD in table 2).

Table 1.Retrieval uncertainty of the PSD parameters in scenarios $C$ and $D$

\begin{tabular}{|c|c|c|c|c|}
\hline Scenario & $r_{\text {eff }}, \%$ & $n, \%$ & $s, \%$ & $v, \%$ \\
\hline C & 120 & 80 & 53 & 155 \\
\hline D & 80 & 75 & 40 & 63 \\
\hline
\end{tabular}

The results in scenario $\mathrm{D}$ shows that the uncertainties we obtained in scenario $\mathrm{C}$ can be reduced if we know the true sphere-spheroid model, i.e. the parameter pair $(\eta, \varepsilon)$. In this case the uncertainties of $r_{\text {eff }}, n, s$ and $v$ decrease to 80, 75,40 and $63 \%$, respectively (see table 1 ). The real parts of the CRI can be estimated with an uncertainty of 0.08 , the imaginary parts with an uncertainty of $i 0.003$ for LA, MA BPSD and $i 0.009$ for HA BPSD (table 2).

\section{CONCLUSIONS}

Preliminary simulations show that the synergy of both instruments allows us to improve the retrieval quality not only of particle size distribution parameters but particularly also allows us to improve the accuracy of the CRI retrievals. If maximum measurement errors of the $3 \beta+2 \alpha+10 \varphi$ data set do not exceed $5 \%$ and if the average uncertain- ty is $2 \%$, the uncertainties of the estimated PSD parameters do not exceed $50 \%$, the accuracy of the CRI retrievals is 0.025 for the real part and $i 0.0025$ for the imaginary part.

Table 2.Retrieval uncertainty of the CRI in scenarios $C$ (numerator) and D (denominator) for MPSD, BPSD and different levels of particle light-absorption

\begin{tabular}{|c|c|c|c|c|}
\hline \multirow{2}{*}{$\begin{array}{c}\text { Particle } \\
\text { Absorption }\end{array}$} & \multicolumn{2}{|c|}{$m_{\mathrm{R}}$} & \multicolumn{2}{|c|}{$m_{\mathrm{I}}$} \\
\hline & MPSD & BPSD & MPSD & BPSD \\
\hline \multirow{2}{*}{ LA } & 0.03 & 0.07 & $i 0.043$ & $i 0.009$ \\
\hline & $\overline{0.07}$ & $\overline{0.04}$ & $\overline{i 0.010}$ & $\overline{i 0.003}$ \\
\hline \multirow{2}{*}{ MA } & 0.07 & 0.07 & $i 0.029$ & $i 0.009$ \\
\hline & $\overline{0.05}$ & $\overline{0.08}$ & $i 0.013$ & $\overline{i 0.003}$ \\
\hline \multirow{2}{*}{ HA } & 0.07 & 0.07 & $i 0.030$ & $i 0.027$ \\
\hline & 0.02 & $\overline{0.08}$ & $\overline{i 0.019}$ & $\overline{i 0.009}$ \\
\hline
\end{tabular}

$10 \%$ measurement error of $3 \beta+2 \alpha$ lidar data can lead to an overestimation of the volume concentration up to $155 \%$ and up to $i 0.03$ of the imaginary part of the CRI. The retrieval results can be stabilized if the true sphere-spheroid model, i.e. the parameter pair $(\eta, \varepsilon)$ is known.

\section{References}

[1] D. Müller, et al., 2013: Vertical profiles of pure dust (SAMUM 2006) and mixed-smoke-dust plumes (SAMUM 2008) inferred from inversion of multiwavelength Raman lidar data and comparison to AERONET retrievals, Appl. Opt. 52 (14), 31783202.

[2] D. Müller, et al., 2014: Airborne multiwavelength High Spectral Resolution Lidar (HSRL-2) observations during TCAP 2012: vertical profiles of optical and microphysical properties of a smoke/urban haze plume over the northeastern coast of the US, Atmos. Meas. Tech. 7, 3487-3496.

[3] I. Veselovskii, et al., 2010: Application of randomly oriented spheroids for retrieval of dust particle parameters from multiwavelength lidar measurements", J.Geophys.Res. 115, D21203.

[4] C. F. Bohren and D. R. Huffman, eds., 1983: Absorption and Scattering of Light by Small Particles, Wiley.

[5] O. Dubovik, et al., 2006: Application of spheroid models to account for aerosol particle nonsphericity in remote sensing of desert dust, J.Geophys.Res. 111, D11208.

[6] A. Kolgotin, et al., 2016: Improved identification of the solution space of aerosol microphysical properties derived from the inversion of profiles of lidar optical data, part 2: simulations with synthetic optical data, Appl. Opt. in press. 